\title{
Atypical Strokes in a Young African American Male: A Case of Mitochondrial Encephalopathy Lactic Acidosis and Stroke-Like Episodes (MELAS) Syndrome
}

\author{
Jully M. Sanchez, Judy Ann Tan, Dimitrios Farmakiotis, and Vikas Aggarwal \\ Department of Internal Medicine, Jacobi Medical Center, Albert Einstein College of Medicine, Bronx, NY, USA \\ Correspondence should be addressed to Vikas Aggarwal, drvikasaggarwal@gmail.com \\ Received 16 February 2011; Accepted 4 May 2011 \\ Academic Editor: Turgut Tatlisumak
}

Copyright ( 2011 Jully M. Sanchez et al. This is an open access article distributed under the Creative Commons Attribution License, which permits unrestricted use, distribution, and reproduction in any medium, provided the original work is properly cited.

Mitochondrial myopathy, encephalopathy, lactic acidosis, and stroke-like episodes (MELAS) syndrome is a rare but important cause of stroke-like symptoms which can often be missed Thambisetty and Newman 2004. We describe a case of a young male presenting with stroke-like episodes, later diagnosed with MELAS in an attempt to improve the understanding about diagnosing MELAS in the appropriate clinical context.

\section{Case Summary}

A 34 year-old black male with no significant past medical history presented with new-onset migraine like headaches and blurry vision. He was ruled out for subarachnoid hemorrhage with a head CT and lumbar puncture and discharged home from the emergency room with analgesics.

He presented again, after 2 months, with left leg weakness and painful cramps. He was found to have left hemiparesis, with spasticity and frequent myoclonic jerks. CT and MR imaging revealed acute right posterior para-sagital temporoparietal ischemic strokes (Figure 1). The rest of the physical exam was normal. His initial blood work (complete blood count, chemistry, coagulation studies) were unremarkable. Further work-up excluded atherosclerosis, hypercoagulable disorders, potential embolic sources, connective tissue and inflammatory or autoimmune disorders. He was soon discharged to a rehabilitation facility and from there to home, with excellent functional improvement.

The patient was re-admitted one month later for sudden onset loss of vision. Physical examination revealed a right homonymous hemianopsia; by that time, his aforementioned left leg hemiparesis was remarkably improved. Neuroimaging on this admission revealed new hypodensities in the left parietal and occipital lobes and near resolution of cerebral lesions noted on his previous admission (Figure 2).

The combination of migraine like headaches, visual complaints, painful myoclonic jerks and rapidly resolving strokes of non-vascular distribution in the posterior areas raised the clinical suspicion of MELAS syndrome. Additional testing revealed a serum lactate of $3.06 \mathrm{mmol} / \mathrm{L}$ (normal: $0.3-1.3 \mathrm{mmol} / \mathrm{L}$ ), CSF lactate of $4.6 \mathrm{mmol} / \mathrm{L}$ (normal: $1.1-$ $2.8 \mathrm{mmol} / \mathrm{L}$ ) and CSF pyruvate of $3.5 \mathrm{mmol} / \mathrm{L}$ (Normal: $0.5-1.7 \mathrm{mmol} / \mathrm{L})$. Genetic testing confirmed the presence of the A-to-G point mutation at the 3243 position in the mitochondrial DNA, which is diagnostic for MELAS [1].

\section{Discussion}

MELAS syndrome is a rare neurodegenerative disease caused by the decreased ability of cells to produce sufficient energy in the form of adenosine $5^{\prime}$-triphosphate [2]. Although it is one of the most common maternally inherited mitochondrial disorders, its exact incidence is unknown [2]. Early development is usually normal and the first symptoms are noted in childhood or early adolescence [3], but the syndrome can present for the first time in older individuals, as demonstrated in the present case. 


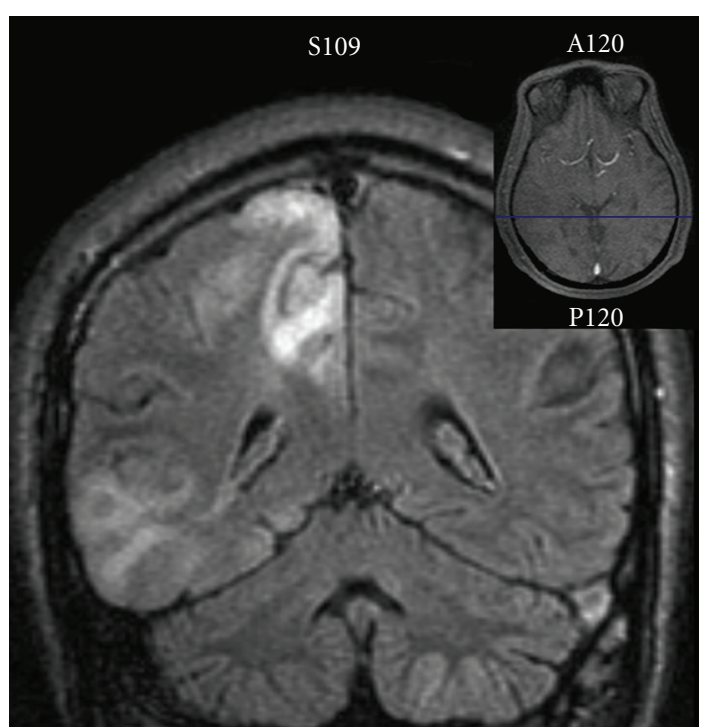

Figure 1: Visit 2 (09/2010) Coronal Flair MRI Image showing acute right para-sagital posterior temporo-parietal ischemic stroke.

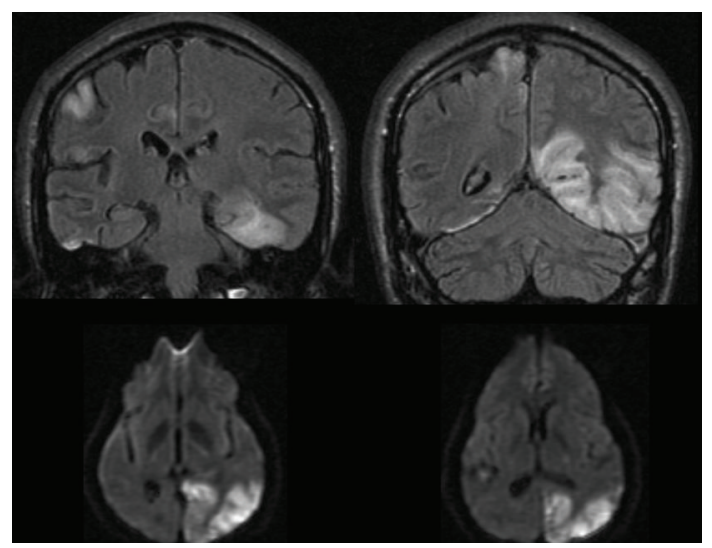

Figure 2: Visit 3 (10/2010) Coronal Flair MRI Image showing hypodensities in the left parietal and occipital lobes and near resolution of cerebral lesions noted on visit 2 .

At least 40 distinct mitochondrial DNA mutations have been associated with MELAS; about $80 \%$ of MELAS patients have an A3243G mutation in the mitochondrial tRNA gene [3]. Exact pathogenesis of the stroke-like episodes remains uncertain, although mitochondrial angiopathy, mitochondrial cytopathy and non-ischemic neurovascular cellular mechanisms have all been suggested [2].

Approximately $80 \%$ of patients with MELAS have symptom onset between the ages of 5 and 15 years. MELAS syndrome has been less frequently described in blacks and the presentation of our patient, except his age and race, was otherwise typical of the syndrome, which is characterized by stroke-like episodes in the parieto-temporal and occipital areas, opthalmoplegia and visual complaints, as well as migraine-type headaches and painful cramps $[2,3]$. Young patients with MELAS can also present with tonic-clonic seizures, hearing loss, as well as autism-like and other psychiatric disorders $[2,3]$.
In addition to its neurologic manifestations, MELAS syndrome may also exhibit multisystem effects including cardiac conduction defects, diabetes mellitus, short stature, myopathy, and gastrointestinal disturbances $[1,2]$. No standard therapies exist for this syndrome but many believe in using l-arginine, coenzyme-Q, B-vitamins, and levocarnitine aiming to minimize the demands on the mitochondria, hence supporting and maximizing their function $[1,2,4]$.

In conclusion, this case underscores the importance of including MELAS in the differential diagnosis of stroke in young male adults, specifically those presenting with ischemic-like strokes in the posterior cerebral areas and with a history of non-specific and easily overlooked symptoms, like recurring headaches, muscle cramps and episodes of blurry vision.

\section{Conflict of Interests}

The authors declare no conflict of interests.

\section{Funding Source}

None needed.

\section{Acknowledgment}

All authors were directly responsible for taking care of the patient and had access to the data and a role in writing the manuscript.

\section{References}

[1] M. Thambisetty and N. J. Newman, "Diagnosis and management of MELAS," Expert Review of Molecular Diagnostics, vol. 4, no. 5, pp. 631-644, 2004.

[2] D. M. Sproule and P. Kaufmann, "Mitochondrial encephalopathy, lactic acidosis, and strokelike episodes: basic concepts, clinical phenotype, and therapeutic management of MELAS syndrome," Annals of the New York Academy of Sciences, vol. 1142, pp. 133-158, 2008.

[3] P. C. Ferrera, C. B. Curran, and H. Swanson, "Etiology of pediatric ischemic stroke," American Journal of Emergency Medicine, vol. 15, no. 7, pp. 671-679, 1997.

[4] F. Scaglia and J. L. Northrop, "The mitochondrial myopathy encephalopathy, lactic acidosis with stroke-like episodes (MELAS) syndrome: a review of treatment options," CNS Drugs, vol. 20, no. 6, pp. 443-464, 2006. 


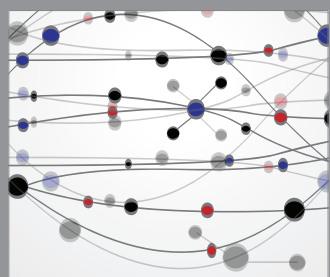

The Scientific World Journal
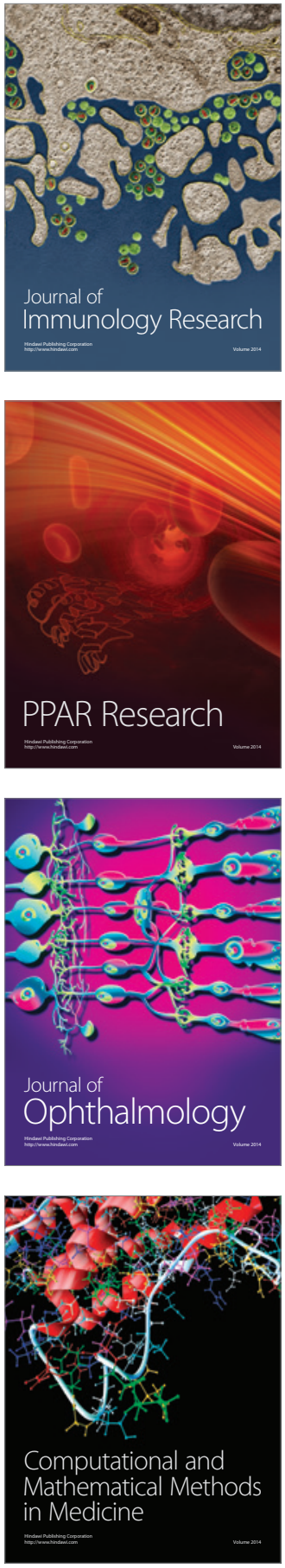

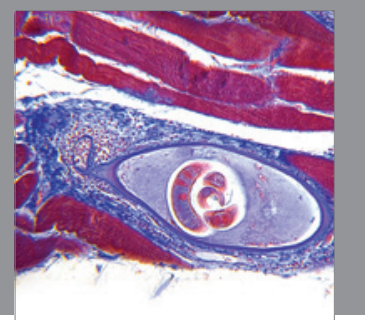

Gastroenterology

Research and Practice
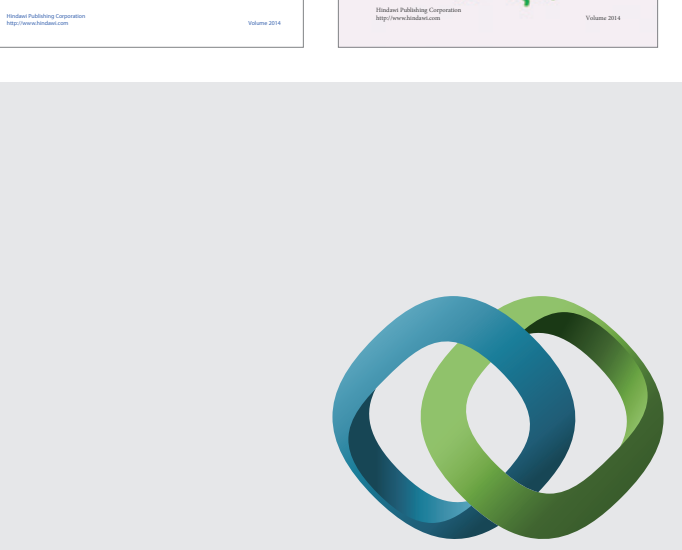

\section{Hindawi}

Submit your manuscripts at

http://www.hindawi.com
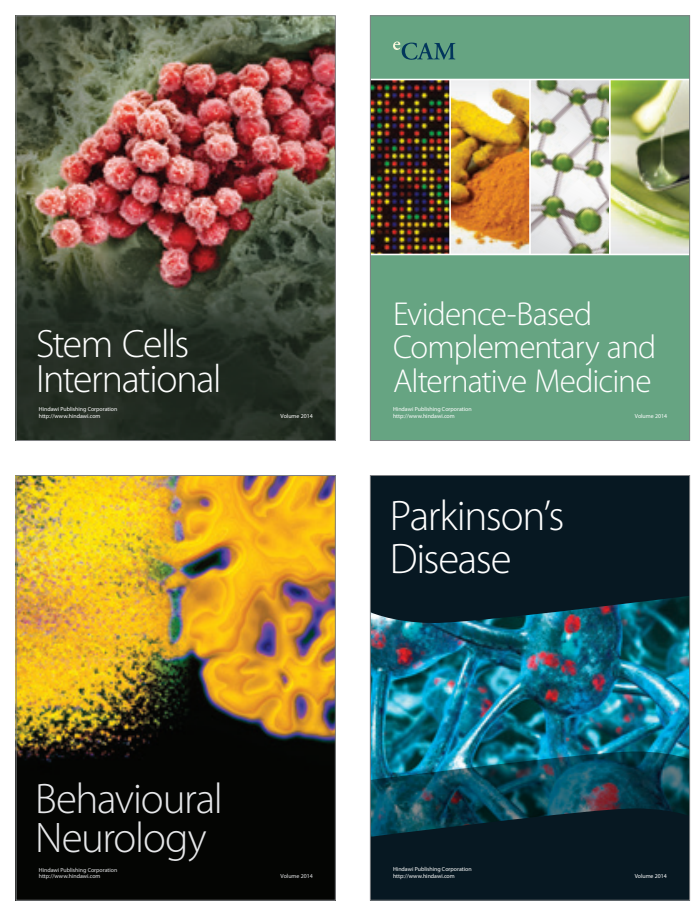

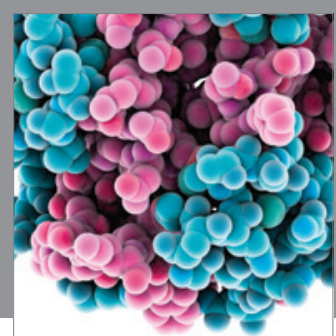

Journal of
Diabetes Research

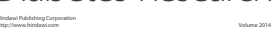

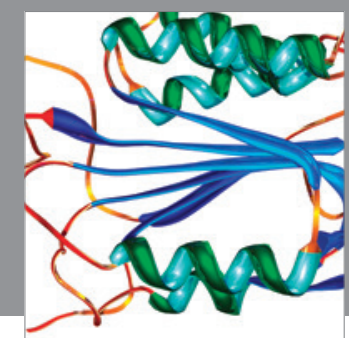

Disease Markers
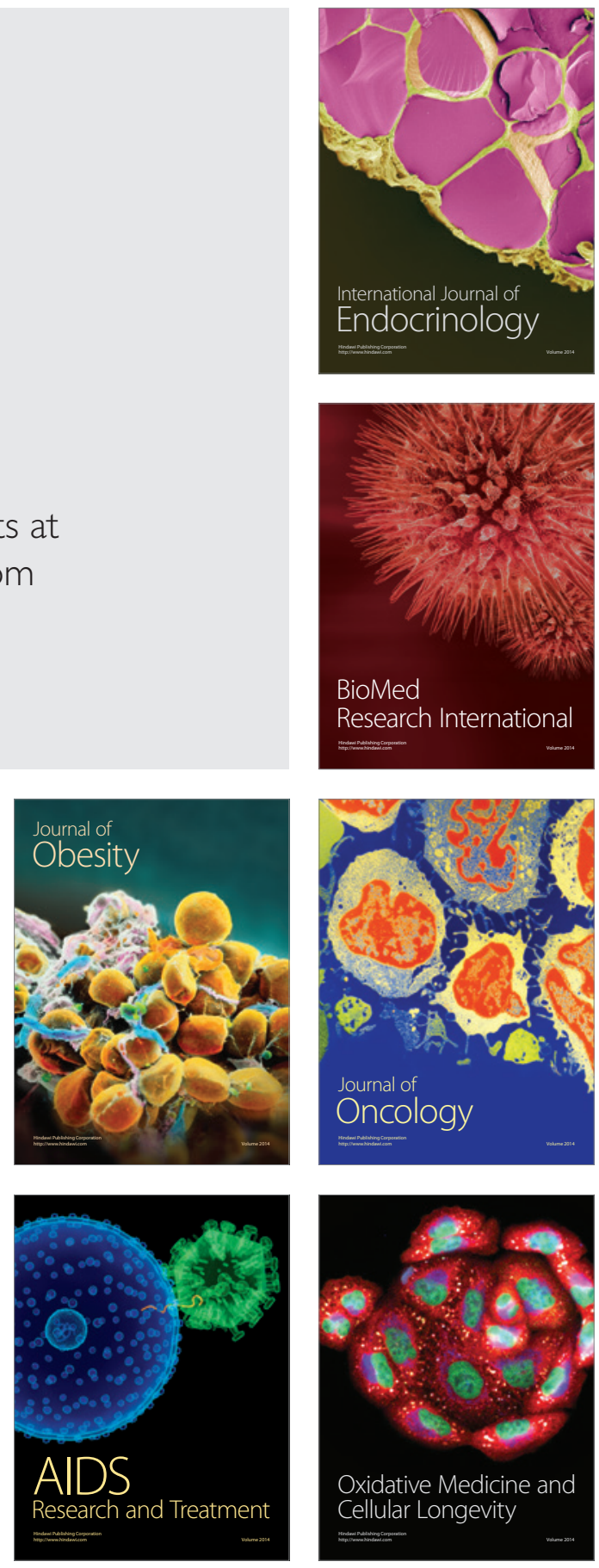